\title{
Distributed Spatial Modulation based Cooperative Diversity Scheme
}

\author{
Quang-Trung Hoang \\ School of Information and Communication Technology \\ Thai Nguyen University, Vietnam \\ hqtrung@ictu.edu.vn
}

\begin{abstract}
In this paper, a distributed spatial modulation based cooperative diversity scheme for relay wireless networks is proposed. Where, the space-time block code is exploited to integrate with distributed spatial modulation. Therefore, the interested transmission scheme achieves high diversity gain. By using Monte-Carlo simulation based on computer, we showed that our proposed transmission scheme outperforms state-of-the-art cooperative relaying schemes in terms bit error rate (BER) performance.
\end{abstract}

KEYWORDS: Space-Time Block Codes, Distributed STBC, Distributed Spatial Modulation, Cooperative Communications, Cooperative Diversity Schemes.

\section{Introduction}

In the future, wireless networks are faced too many user demands for high rate and low delay transmission. Especially, modern mobile devices are rapidly increasing over each year. This requires next mobile communication systems as $5 \mathrm{G}$ to overcome challenges of technologies. Among other solutions, cooperative communication technique is an expected solution, and is considered more by the research community, recently.

With cooperative wireless networks, mobile users are not only able to make direct connectivities, as device-to-device (D2D) communications in [1], but they also can use mediate relays for collaboration to forwarding data from source to destination. Further, since the cooperative communication technique is combined with some techniques as network coding $(\mathrm{NC})$ or space-time block code (STBC) and spatial modulation (SM), the performance of wireless networks is significantly improved.

Distributed space-time block code (D-STBC) is an STBC technique applied for distributed wireless networks to support diversity gain similar to the multi-input multi-output (MIMO) systems. In reference [2], Bansal et al. showed the efficiency of Alamouti typed D-STBC when it is used for cooperative relaying schemes. In addition, limitations on transmission delay are also overcome by physical layer network coding [3]. This enables cooperative relaying networks to increase the high throughput in case there exists bidirectional traffic that is necessary for widespread information exchange in future wireless services.

The last years, the spatial modulation, also known as a new technique, is interested in more-and-more researchers. This method uses two constellations, one for signal modulation and another one for selecting antennas to transmit modulated signals. In MIMO systems, the technique SM is proved that it can highly increase the energy efficiency of a system [4]. By exploiting capabilities of SM to apply for distributed wireless networks, the system performance will be enhanced, and SM is then called DSM (Distributed SM). 
In references [5]-[6], authors investigated cooperative transmission schemes based on DSM, and showed that DSM enables to achieve high diversity gain being closed to the case of ones without using DSM. Further, this technique can also reduce transmission energy. However, researchers in [5]-[6] still omit some problems that are necessary to continue researching more: The first, authors in [5] just consider in relaying transmission without cooperation among relays or between a source and any relay to forward data, simultaneously. In this case, the transmission scheme can achieve the gain of DSM but not spatial diversity gain. The second, although the DSM-STBC protocol in [7] achieves both advantage of DSM and diversity gain of distributed Alamouti-STBC, the performance of this protocol is significantly affected by imperfect demodulation of two relays chosen.

To deal with the above problems, in this paper, we propose a DSM based cooperative diversity scheme, so-called the DSM-CD scheme. Where, we consider in keeping both spatial diversity and modulation gain for enhancing the system performance in terms BER. By letting the source node cooperate with the relay node in data forwarding phase ${ }^{1}$, the proposed scheme will obtain better spatial diversity gain than the protocol in [5]. Further, thank using collaboration between the source node and one of the relay nodes instead of transmission cooperation the among only relays, our DSM-CD scheme reduces the amount of errors occurred by relays. The reason is that the source plays a role as one relay node in the data forwarding phase (the second phase), so there is just one relay which must decode the source'data, and a number of decoding errors occurred at relays are decreased. Then, the destination node can decode data better than the case of protocol in [7] over considered SNR ranges.

Our contributions include as follows:

- The DSM-CD scheme, which achieves two advantages: (i) the Alamouti distributed STBC is integrated with DSM to enhance diversity gain; (ii) the cooperative mechanism between the source node and the activated relay, which significantly decreases errors occurred by the distributed spatial modulation, and the BER performance of the system is improved.

- The Monte-Carlo simulations are used to show that our proposal outperforms state-ofthe-art cooperative diversity schemes.

The remainder of this paper is organized as follows. In Section 2, the considered system model is introduced. Next, the proposed DSM-CD scheme is studied in Section 3. In Section 4 , simulation results and evaluations are commented. Finally, Section 5 shows the conclusion of this paper.

\section{System model}

For simply, the considered system model of cooperative wireless networks is shown as in Figure 1. Where, it consists of one source (S), one destination (D) and two relays $\left(R_{k}\right.$, with $k=1,2$ ). Assume that each network node is equipped only one single antenna and works in the half-duplex mode. The process of cooperative transmission is implemented in two phases: (i) in the first phase, the source broadcasts its data to both the destination and

\footnotetext{
${ }^{1}$ Cooperative transmission is implemented in two phases: the first phase, the source node broadcasts its data to relays. In the second phase, relay nodes forward source'data to the destination node.
} 


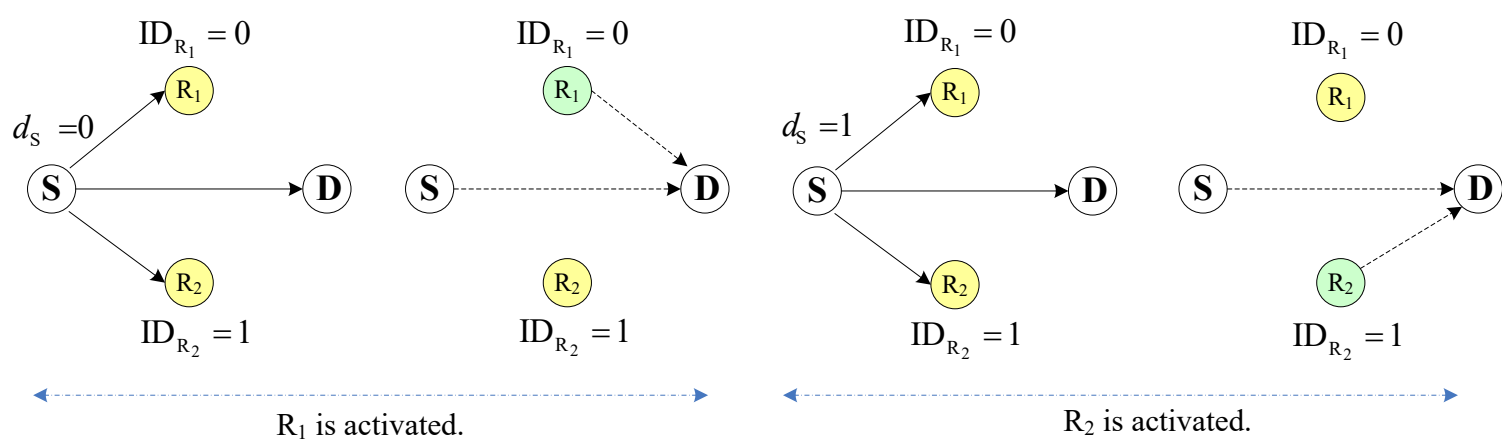

Fig. 1. The DSM-CD scheme with a source, a destination and two relays.

relays nodes; (ii) in the second phase, one of two relays collaborates with the source to forward data block of Alamouti STBC to the destination node. With the interested model, the distributed spatial modulation [5] is used at the relays, so the cooperative diversity scheme can alternatively switch on between two relays. At each instant time, the activated relay will participate cooperative transmission process while the rest one (being not activated) keeps silent.

The channel between node $\mathrm{X}$ and $\mathrm{Y}$ is affected by Rayleigh fading, with a gain $h_{X Y}$. The noise occurred at receiver of each network node is a complex Additive White Gaussian random variable with zero mean and variance $\sigma_{n}^{2}=N_{0} / 2$ per dimension.

Assume that, the source transmits Phase Shift Keying (PSK) or Quadrature Amplitude Modulation (QAM) symbols belonging to constellation $A$ with size $M$ while the relays use PSK/QAM modulation with constellation size $N$. Because DSM is used at the relays, value $M$ and $N$ can be different. Where, the number of potential relays used for cooperative transmission is $N_{r} \geq M$. In case $N_{r}=2$, as the considered model, the constellation size is $M=N=2$. In addition, each relay is assigned a unique identify $\mathbf{I D}_{R_{k}}$. With two cooperative relays, $k=1,2$. For example, $\mathbf{I D}_{R_{1}}=0$ and $\mathbf{I D}_{R_{2}}=1$.

\section{Proposed DSM-CD Scheme}

In this section, we detail the operation of DSM-CD scheme. Where, we also use the distributed spatial modulation (DSM) proposed in [5]. However, the different point between our scheme and [5] is that we let the source cooperate with the relay to transmit data simultaneously in the relaying phase while the source using protocol proposed in [5] keeps silent. By the way, our DSM-CD scheme does not only achieve high diversity but it also decreases the computing complex of the demodulator at the destination node.

\subsection{Broadcasting phase}

Assuming the source needs to send three data symbols $d_{1}, d_{2}$ and $d_{3}$ to the destination. With $N_{r}=2$, we use PSK modulation for both the source and relays, so $M=N=2$. Then, modulated symbols are $s_{1}, s_{2}$ and $s_{3}$, respectively. In the broadcasting phase, the source sequentially transmits these symbols to both the destination and relays. Therefore, signals received at the relays are determined as follows:

$$
y_{t}^{S R_{k}}=\sqrt{E_{1}^{S}} h_{S R_{k}} s_{t}+n_{t}^{R_{k}}
$$


where, $y_{t}^{S R_{k}}$ is signal received at the $k^{\text {th }}$ relay $(k=1,2)$, at time-slots $t=1,2,3 ; E_{1}^{S}$ is the source's transmit energy during the first phase; $h_{S R_{k}}$ is the channel coefficient between the source and $k^{\text {th }}$ relay, which is assumed to be constant over the transmission of $s_{1}, s_{2}$ and $s_{3} ; n_{t}^{R_{k}}$ is the Adaptive White Gauss Noise (AWGN) element.

Similarly, the signals received at the destination node in the broadcasting phase (the first phase) are written by:

$$
y_{t}^{S D_{1}}=\sqrt{E_{1}^{S}} h_{S D} s_{t}+n_{t}^{D_{1}} .
$$

The signal $y_{t}^{S D_{1}}$ is used to provide information for demodulation process at the destination node. In order to demodulate the source's signals, the Maximal-Likelihood (ML) criterion is used. Then, the demodulated signals are written as follows:

$$
\hat{s}_{t}^{S R_{k}}=\arg \min _{\tilde{s}_{t}^{S R_{k}} \in A}\left\{\left|y_{t}^{S R_{k}}-\sqrt{E_{1}^{S}} h_{S R_{k}} \tilde{s}_{t}^{S R_{k}}\right|^{2}\right\}
$$

Thus, the estimated data symbols $d_{t}^{\hat{S R} R_{k}}$ are formulated by $\hat{d}_{t}^{S R_{k}}=\Phi_{m}\left(\hat{s}_{t}^{S R_{k}}\right)$. Where, $\Phi_{m}(\cdot)$ is the PSK modulation - to - bit mapping function.

\subsection{Relaying phase}

After estimating the source's data symbols, each $k^{\text {th }}$ relay will be activated to collaborate with the source if its $\mathbf{I D}_{R_{k}}$ and $d_{1}^{S R_{k}}$ is coincident. Then, a pair of this relay and the source transmit Alamouti STBC formed by

$$
\left[\begin{array}{cc}
\hat{s}_{2}^{S R_{a}} & s_{3} \\
\left(-\hat{s}_{3}^{S R_{a}}\right)^{*} & \left(s_{2}\right)^{*}
\end{array}\right]
$$

where, $\hat{s}_{t}^{S R_{a}},(t=2,3)$, is the activated relay's modulation symbols estimated from the source's signals; $s_{2}$ and $s_{3}$ are the modulated signals of $d_{2}$ and $d_{3}$, respectively. Thus, although the source plays a role as one relay but it just transmits the original data symbols without the estimated ones. By this way, the signals received at the destination, during two consecutive time slots of the relaying phase, are formulated as follows:

$$
\begin{aligned}
& y_{1}^{D}=\sqrt{E_{R}} h_{R_{a} D} \hat{s}_{2}^{S R_{a}}+\sqrt{E_{2}^{S}} h_{S D} s_{3}+n_{1}^{D} \\
& y_{2}^{D}=\sqrt{E_{R}} h_{R_{a} D}\left(-\hat{s}_{3}^{S R_{a}}\right)^{*}+\sqrt{E_{2}^{S}} h_{S D}\left(s_{2}\right)^{*}+n_{2}^{D}
\end{aligned}
$$

Where, $E_{R}$ is the activated relay's transmit energy and $E_{2}^{S}$ is the source's transmit energy during the relaying phase.

\subsection{Demodulator at The Destination}

In this paper, we adopt the error-aware ML demodulator in [7] for detecting the source's data from the distributed Alamouti STBC received at the destination. However, because one of two cooperating relays forwarding the source's data is just the source, so the number of errors occurred by the distributed spatial modulation (DSM) depends on only one rest 
relay. The data symbols demodulated at the destination node are formulated by (7) and (8). Where, $\hat{s}_{t}^{S R_{a}}, t=\{2,3\}$, are the modulation symbols of the activated relay.

$$
\begin{aligned}
& {\left[\hat{\mathbf{s}}^{D}\right]=\arg \max _{\tilde{s}_{t}^{D} \in A, t=1,2,3}} \\
& \left\{\sum_{\tilde{s}_{1}^{D} \in A} \sum_{\tilde{s}_{2}^{D} \in A} \sum_{\tilde{s}_{3}^{D} \in A} \sum_{\hat{s}_{1}^{S R_{1}} \in A} \sum_{\hat{s}_{2}^{S R_{1}} \in A} \sum_{\hat{s}_{3}^{S R_{1}} \in A} \sum_{\hat{s}_{1}^{S R_{2}} \in A} \sum_{\hat{s}_{2}^{S R_{2}} \in A} \sum_{\hat{s}_{3}^{S R_{2}} \in A} \operatorname{Pr}\left\{\left[y_{1}^{D}, y_{2}^{D}\right] \mid\left(\tilde{\mathbf{s}}^{D}, \hat{\mathbf{s}}^{S R}\right)\right\} \operatorname{Pr}\left\{\tilde{\mathbf{s}}^{D}, \hat{\mathbf{s}}^{S R}\right\}\right\} \\
& \operatorname{Pr}\left\{\left[y_{1}^{D}, y_{2}^{D}\right] \mid\left(\tilde{\mathbf{s}}^{D}, \hat{\mathbf{s}}^{S R}\right)\right\}=\exp \left(\frac{y_{1}^{D}-\left(\sqrt{E_{R}} h_{R_{a} D} \hat{s}_{2}^{S R_{a}}+\sqrt{E_{2}^{S}} h_{S D} s_{3}\right)}{N_{0}}\right) \\
& \times \exp \left(\frac{y_{2}^{D}-\left(\sqrt{E_{R}} h_{R_{a} D}\left(-\hat{s}_{3}^{S R_{a}}\right)^{*}+\sqrt{E_{2}^{S}} h_{S D}\left(s_{2}\right)^{*}\right)}{N_{0}}\right)
\end{aligned}
$$

Where, $\left[\hat{\mathbf{s}}^{D}\right]=\left[s_{1}^{D}, s_{2}^{D}, s_{3}^{D}\right]$ presents the estimated data vector of the source at the destination; $\left[\tilde{\mathbf{s}}^{D}\right]=\left[\tilde{s}_{1}^{D}, \tilde{s}_{2}^{D}, \tilde{s}_{3}^{D}\right]$ is the $1 \times 3$ trial data vector at the destination for the hypothesis detection problem; $\hat{\mathbf{s}}^{S R}=\left[s_{1}^{S R_{1}}, s_{2}^{S R_{1}}, s_{3}^{S R_{1}}, s_{1}^{S R_{2}}, s_{2}^{S R_{2}}, s_{3}^{S R_{2}}\right]$ denotes the 1x6 demodulated data vector at the relays; $\operatorname{Pr}\left\{\tilde{\mathbf{s}}^{D}, \hat{\mathbf{s}}^{S R}\right\}$ is the priori joint probability of the demodulation errors at the relays. At high SNR values, the ML demodulator in (7) is rewritten as follows:

$$
\left[\hat{\mathbf{s}}^{D}\right]=\arg \min _{\tilde{s}_{t}^{D} \in A, t=1,2,3}\left\{\Lambda^{h i g h-s n r}\left(y_{1}^{D}, y_{2}^{D}, \tilde{\mathbf{s}}^{D}, \hat{\mathbf{s}}^{S R}\right)\right\}
$$

where, $\Lambda^{h i g h-s n r}\left(y_{1}^{D}, y_{2}^{D}, \tilde{\mathbf{s}}^{D}, \hat{\mathbf{s}}^{S R}\right)$ is determined as in the formulation (10).

$$
\begin{aligned}
& \Lambda^{h i g h-s n r}\left(y_{1}^{D}, y_{2}^{D}, \tilde{\mathbf{s}}^{D}, \hat{\mathbf{s}}^{S R}\right)=Q+\left|y_{1}^{D}-\left(\sqrt{E_{R}} h_{R_{a} D} \hat{s}_{2}^{S R_{a}}+\sqrt{E_{2}^{S}} h_{S D} s_{3}\right)\right| \\
& +\left|y_{2}^{D}-\left(\sqrt{E_{R}} h_{R_{a} D}\left(-\hat{s}_{3}^{S R_{a}}\right)^{*}+\sqrt{E_{2}^{S}} h_{S D}\left(s_{2}\right)^{*}\right)\right| \\
& +\sum_{k=1}^{2} \sum_{t=1}^{3}\left(E_{1}^{S}\left|h^{S R_{k}}\right|^{2}\left|\tilde{s}_{t}^{D}-\hat{s}_{t}^{S R_{k}}\right|^{2}\right) \text {. } \\
& Q=\left|y_{1}^{S D}-\sqrt{E_{1}^{S}} h_{S D} s_{1}\right|^{2}+\left|y_{2}^{S D}-\sqrt{E_{1}^{S}} h_{S D} s_{2}\right|^{2}+\left|y_{3}^{S D}-\sqrt{E_{1}^{S}} h_{S D} s_{3}\right|^{2}
\end{aligned}
$$

Observe the formulations (7), (9) and (10), clearly by using our proposed DSM-CD scheme the demodulator at destination can reduce a number of cooperating relays while the distributed Alamouti STBC is still transmitted.

\section{Simulation Results}

In order to evaluate the performance of proposed scheme, we use Monte-Carlo simulation based on computer for the network model as in Figure 1. Where, the links between two 


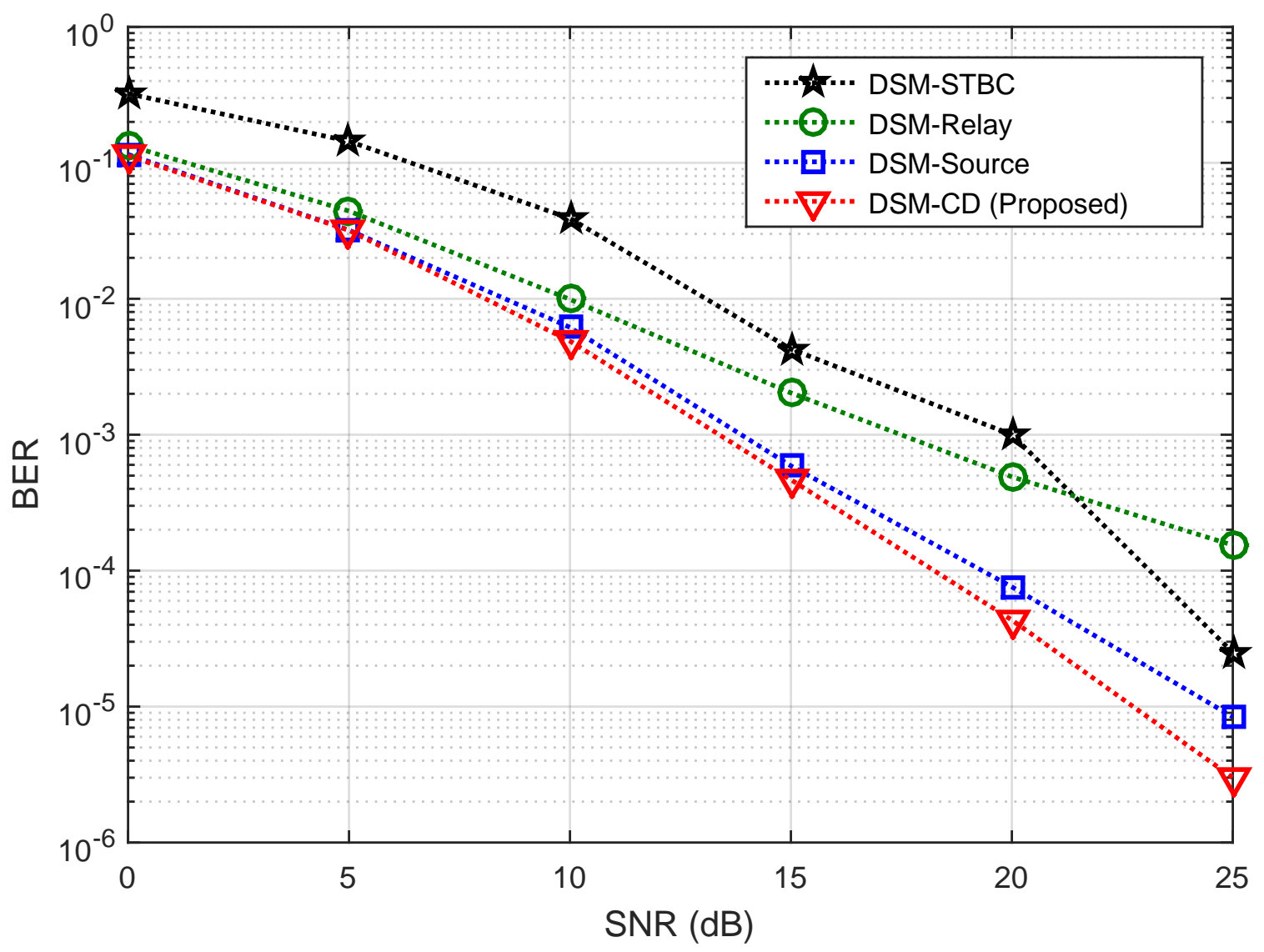

Fig. 2. The BER performance of the proposed DSM-CD scheme.

any nodes are the Rayleigh fading channels, with variance $\sigma^{2}=\sigma_{S D}^{2}=\sigma_{S R}^{2}=\sigma_{R D}^{2}=1$ and zero mean. The noise at receivers is AWGN with zero and variance $\sigma_{n}^{2}=N_{0} / 2$. We assume the average transmit energy per symbol is $E$. Thus, in the second transmission phase, the source transmits symbol with the energy $E_{2}^{S}$ and the activated relay transmits symbol with the energy $E_{R}, E_{2}^{S}=E-E_{R}$. Further, the PSK modulation is used. The simulation is implemented to investigate the BER performance of the transmission schemes over the common $\mathrm{SNR}$ ranges $\left(\mathrm{SNR}_{\mathrm{SD}}=\mathrm{SNR}_{\mathrm{SR}}=\mathrm{SNR}_{\mathrm{RD}}=\mathrm{SNR}\right)$. Then, the BER performance of the DSM-CD scheme is compared with schemes in [5] and [7]. Simulation results are shown as in Figure 2. Clearly, the DSM-CD scheme obtains the better BER performance than the others. can be explained as follows. With the DSM scheme proposed in [5], the distribution Alamouti STBC is not used, so in the second transmission phase the diversity gain is not achieved. More especially, if let relays transmit their symbols estimated from the source (case of DSM-Relay), errors occurred at the destination will be increased. Thus, the BER performance is low. However, if let the relays transmit the source's original data symbols (case of DSM-Source), the diversity gain will be highly increased. In fact, the demodulation of relays is imperfect. The DSM-CD scheme allows the error-aware ML demodulator of destination combine information of both the source and relays, so the high diversity is achieved.

Compared to the DSM-STBC scheme in [7], our proposed DSM-CD scheme reduces a number of relays demodulating the source's data symbols. Thus, the errors occurred by the relays used DSM is decreased. In contrast, the demodulation errors at relays make the 
DSM-STBC scheme in [7] decrease the diversity gain. At the considered SNR ranges, our DSM-CD scheme also outperforms the DSM-STBC scheme in terms the BER performance. In the cooperative networks, the network nodes always get opportunities to collaborate with each other for increasing the transmission rate. Thus, even if there exist good links, each node still needs to cooperate with any node to obtain the best network performance.

\section{Conclusion}

In this paper, we proposed the DSM-CD scheme for the cooperative wireless networks. Our proposal can deal disadvantages of the state-of-the-art schemes on the diversity gains as well as the system complex. Especially, the proposed cooperative diversity scheme in this paper can be well applied for wireless ad-hoc and sensor networks, which are considered more in the future wireless network designs. In the next research, we will consider in the cross-layer design consisted of MAC layer.

\section{Acknowledgment}

This paper was supported by the School of Information and Communication Technology Thai Nguyen University in Vietnam under Project: T2017-07-17.

\section{References}

1. E. Datsika, A. Antonopoulos, N. Zorba, and C. Verikoukis, "Green cooperative device - to - device communication: a social - aware perspective," IEEE Access, vol. 4, pp. 3697-3707, 2016.

2. A. Bansal, M. R. Bhatnagar, and A. Hjorungnes, "Decoding and performance bound of demodulateand-forward based distributed alamouti stbc," IEEE Transactions on Wireless Communications, vol. 12, pp. 702-713, February 2013.

3. S. Zhang, S. C. Liew, and P. P. Lam, "Hot topic: Physical-layer network coding," in Proceedings of the 12th Annual International Conference on Mobile Computing and Networking, MobiCom '06, (New York, NY, USA), pp. 358-365, ACM, 2006.

4. M. D. Renzo, H. Haas, A. Ghrayeb, S. Sugiura, and L. Hanzo, "Spatial modulation for generalized mimo: Challenges, opportunities, and implementation," Proceedings of the IEEE, vol. 102, pp. 56-103, Jan 2014.

5. S. Narayanan, M. D. Renzo, F. Graziosi, and H. Haas, "Distributed spatial modulation for relay networks," in Vehicular Technology Conference (VTC Fall), 2013 IEEE 78th, pp. 1-6, Sept 2013.

6. S. Narayanan, M. D. Renzo, F. Graziosi, and H. Haas, "Distributed spatial modulation: A cooperative diversity protocol for half-duplex relay-aided wireless networks," IEEE Transactions on Vehicular Technology, vol. 65, pp. 2947-2964, May 2016.

7. S. Narayanan, A. Stavridis, M. D. Renzo, F. Graziosi, and H. Haas, "Distributed spatially-modulated space-time-block-codes," in 2013 IEEE 18th International Workshop on Computer Aided Modeling and Design of Communication Links and Networks (CAMAD), pp. 159-163, Sept 2013. 